\title{
As Integrais de Mellin-Barnes e a Função de Fox
}

\author{
F. SILVA COSTA ${ }^{1}$, J. VAZ JR. ${ }^{2}$ E. CAPELAS DE OLIVEIRA ${ }^{3}$, Departamento de \\ Matemática Aplicada - Imecc - Unicamp, 13083-859 Campinas, SP, Brasil. \\ R. FIGUEIREDO CAMARGO ${ }^{4}$, Faculdade de Ciências - Unesp, 17033-360 \\ Bauru, SP, Brasil.
}

Resumo. A partir do conceito de integrais de Mellin-Barnes, apresentamos a função de Fox e algumas de suas propriedades a fim de discutir a equação diferencial fracionária associada ao problema do telégrafo.

Palavras-chave. Integral de Mellin-Barnes, função de Fox, função de Meijer, função hipergeométrica, equação do telégrafo fracionária.

\section{Introdução}

Chama-se função especial a toda função que, em geral, pode ser inserida numa particular classe de funções. Por exemplo, a função de Legendre é um caso particular da clássica função hipergeométrica que, por sua vez, é um caso particular da classe das funções hipergeométricas. Em completa analogia, a função de Bessel é um caso particular da função hipergeométrica confluente a qual é obtida, através de um conveniente processo de limite, a partir da clássica função hipergeométrica [3].

São várias as maneiras de se efetuar um estudo sistemático envolvendo as funções especiais das quais podemos mencionar: (a) a partir de uma função geratriz [14]; (b) utilizando relações de recorrência [32]; (c) usando a respectiva equação diferencial ordinária, quando pertinente ${ }^{5}$, bem como (d) a partir de representações integrais [36], dentre outras. Visto que colocamos uma restrição (pertinência) podemos formular a pergunta: Existe uma maneira geral de se abordar as funções especiais?

Antes de respondermos a esta pergunta, vamos nos restringir à classe de funções especiais contendo apenas uma variável independente. ${ }^{6}$ Agora sim, neste caso, uma maneira conveniente de abordar este estudo é através das chamadas integrais (no plano complexo) de Mellin-Barnes, conforme introduzidas, num trabalho pioneiro,

\footnotetext{
${ }^{1}$ pdiulfelix@hotmail.com

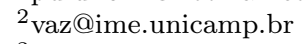

${ }^{3}$ capelas@ime.unicamp.br

${ }^{4}$ rubens@fc.unesp.br

${ }^{5}$ Existem funções especiais que não satisfazem a uma específica equação diferencial, dentre elas, mencionamos a função $H$ de Fox.

${ }^{6}$ Existem funções especiais que dependem de mais de uma variável independente, bem como de mais de um parâmetro. Apenas para citar, as funções de Lauricella pertencem a esta classe [18].
} 
por Pincherle [29] e desenvolvida a teoria por Mellin e, nas aplicações, por Barnes que, por isso, hoje, justificam o nome de integrais de Mellin-Barnes. ${ }^{7}$ Estas integrais se caracterizam por conterem no integrando, de um modo geral, um quociente entre produtos de funções gama. Como complemento à resposta da pergunta, aqui, vamos considerar a classe de funções conhecida pelo nome de função $H$ de Fox [13]. Esta função admite um importante caso especial, a chamada função $G$ de Meijer [27], que por sua vez congrega, obtidas como casos particulares, todas as clássicas funções especiais da Física-Matemática, dentre elas as funções hipergeométricas [17].

A fim de justificar a importância do estudo das funções de Fox e suas aplicações mencionamos alguns recentes trabalhos onde tal função emerge naturalmente, a saber: no estudo da equação de Schrödinger com potenciais tipo delta [4]; no tunelamento [5]; no estudo de espalhamento [28]; equação de difusão não Markoviana [19]; dentre outros [26].

Em resumo, vamos apresentar a função $H$ de Fox através das integrais de MellinBarnes e discutir algumas propriedades a fim de, como aplicação, resolver a equação diferencial fracionária associada ao problema do telégrafo e recuperar, como caso particular, um recente resultado [35].

Este trabalho está disposto da seguinte maneira: Na Seção 2, introduzimos as integrais de Mellin-Barnes exemplificando a sua utilidade no caso da clássica função hipergeométrica. Na Seção 3, apresentamos a função $H$ de Fox e mencionamos apenas as propriedades que serão utilizadas no decorrer do trabalho, especificamente na Seção 5. Na Seção 4 efetuamos um resumo das preliminares envolvendo o cálculo fracionário, em particular as transformadas de Laplace e Fourier a fim de resolver, na Seção 5, o problema do telégrafo na versão fracionária, isto é, a equação diferencial parcial fracionária associada ao problema do telégrafo.

\section{As Integrais de Mellin-Barnes}

Em um recente artigo Mainardi-Pagnini [23] recuperaram os resultados de Pincherle [29] onde foi introduzido, pela primeira vez, a partir do chamado "princípio da dualidade", as hoje conhecidas como integrais de Mellin-Barnes ${ }^{8}$. Também recente é o artigo de Saxena [33], onde é apresentado um resumo da vida acadêmica de Fox, em particular como foi introduzida a função $H$ de Fox em termos das integrais de Mellin-Barnes. Infelizmente, no artigo de Saxena não é feita nenhuma menção ao artigo de Mainardi-Pagnini, apesar de terem sido dados créditos aos trabalhos de Pincherle do ano de 1888.

Em analogia às transformadas de Laplace e de Fourier, podemos introduzir a transformada de Mellin através de um par de integrais, isto é, a transformada de Mellin direta e a respectiva transformada de Mellin inversa. No caso particular de a função a ser transformada conter uma ou mais funções gama, vamos obter

\footnotetext{
${ }^{7}$ De todas as integrais que contêm funções gama em seus integrandos, as mais importantes são as assim chamadas integrais de Mellin-Barnes. Tais integrais foram introduzidas por Pincherle em 1888; sua teoria foi desenvolvida, em 1910, por Mellin e foram usadas por Barnes na integração completa da equação hipergeométrica. Tradução livre [9].

${ }^{8}$ São dois trabalhos que constam do Volume I delle Opere Scelte, Unione Matematica Italiana, Edizioni Cremonese, páginas 223-230 e 231-239, (1954), respectivamente.
} 
as chamadas integrais de Mellin-Barnes. Tais integrais desempenham um papel fundamental, em particular, como vamos ver na Seção 3, no estudo das funções $H$ de Fox que, como já afirmamos, contêm, como casos particulares, todas as clássicas funções especiais da Física-Matemática e os vários tipos de funções de Mittag-Leffler e a chamada função de Wright-Fox [21], dentre outras.

Aqui, por entendermos que as transformadas integrais desempenham um papel importante quando da resolução de um problema envolvendo uma equação diferencial, ordinária ou parcial, e condições iniciais/contorno, vamos introduzir as integrais de Mellin-Barnes através de uma conveniente transformada inversa, em particular, a transformada de Mellin.

Para tal, introduzimos, na forma de um teorema, o par de transformadas de Mellin, ou seja, a transformada direta e a respectiva transformada inversa.

Teorema 2.1 (Transformada de Mellin). Considere $f(x)$ uma função tal que a integral $\int_{0}^{\infty} x^{k-1}|f(x)| \mathrm{d} x$ seja limitada para algum $k>0$. Se a transformada de Mellin é dada pela integral

$$
\mathfrak{M}[f(x)] \equiv F(s)=\int_{0}^{\infty} x^{s-1} f(x) \mathrm{d} x,
$$

então a transformada de Mellin inversa é dada por [34]

$$
\mathfrak{M}^{-1}[F(s)] \equiv f(x)=\frac{1}{2 \pi i} \int_{c-i \infty}^{c+\infty} F(s) x^{-s} \mathrm{~d} s,
$$

onde $c>k$.

Chama-se integral de Mellin-Barnes a toda integral no plano complexo cujo integrando contempla pelo menos uma função gama. Convém ressaltar que Pincherle [29] obteve a seguinte fórmula

$$
\Psi(t)=\frac{1}{2 \pi i} \int_{a-i \infty}^{a+i \infty} \frac{\prod_{i=1}^{m} \Gamma\left(x-\rho_{i}\right)}{\prod_{i=1}^{m-1} \Gamma\left(x-\sigma_{i}\right)} \mathrm{e}^{x t} \mathrm{~d} x
$$

com $a>\operatorname{Re}\left\{\rho_{1}, \rho_{2}, \ldots, \rho_{m}\right\}$, cuja convergência foi provada usando uma fórmula assintótica para a função gama. Esta integral pode ser considerada como o primeiro exemplo na literatura do que é hoje conhecido com o nome de integral de MellinBarnes [23].

\subsection{Função Hipergeométrica}

A fim de exemplificarmos o cálculo explícito de uma transformada de Mellin, vamos obter a transformada de Mellin da função hipergeométrica, ou seja, calcular a seguinte integral

$$
\mathfrak{M}\left[{ }_{2} F_{1}(a, b ; c ;-x)\right]=\int_{0}^{\infty} x^{s-1}{ }_{2} F_{1}(a, b ; c ;-x) \mathrm{d} x,
$$


onde $a, b, c \in \mathbb{C}$ e $\operatorname{Re}(s)>0$.

Para calcular esta integral, utilizamos a representação integral devida a Euler, já invertendo a ordem das integrações, de modo a obtermos

$$
\mathfrak{M}\left[{ }_{2} F_{1}(a, b ; c ;-x)\right]=\frac{\Gamma(c)}{\Gamma(b) \Gamma(c-b)} \int_{0}^{1} \xi^{b-1}(1-\xi)^{c-b-1} \mathrm{~d} \xi \cdot \int_{0}^{\infty} \frac{x^{s-1}}{(1+\xi x)^{a}} \mathrm{~d} x .
$$

Primeiramente, introduzimos a mudança de variável $x=(1-t) / t \xi$ cuja integral na variável $t$ é dada em termos de uma função beta. Enfim, novamente utilizando a definição da função beta para integrar na variável $\xi$, obtemos

$$
\mathfrak{M}\left[{ }_{2} F_{1}(a, b ; c ;-x)\right]=\frac{\Gamma(c) \Gamma(s) \Gamma(a-s) \Gamma(b-s)}{\Gamma(a) \Gamma(b) \Gamma(c-s)},
$$

para $\operatorname{Re}(s)>0, \operatorname{Re}(a-s)>0, \operatorname{Re}(b-s)>0$ e $\operatorname{Re}(c-s)>0$.

A partir do teorema envolvendo o par de transformadas de Mellin podemos escrever a respectiva transformada de Mellin inversa

$$
\mathfrak{M}^{-1}\left[\frac{\Gamma(c) \Gamma(s) \Gamma(a-s) \Gamma(b-s)}{\Gamma(a) \Gamma(b) \Gamma(c-s)}\right]={ }_{2} F_{1}(a, b ; c ;-x) .
$$

Enfim, introduzindo a integral da transformada inversa podemos escrever a seguinte representação integral, no plano complexo, para a clássica função hipergeométrica

$$
{ }_{2} F_{1}(a, b ; c ; z)=\frac{\Gamma(c)}{\Gamma(a) \Gamma(b)} \frac{1}{2 \pi i} \int_{\gamma-i \infty}^{\gamma+i \infty} \frac{\Gamma(s) \Gamma(a-s) \Gamma(b-s)}{\Gamma(c-s)}(-z)^{-s} \mathrm{~d} s,
$$

onde $a, b, c \in \mathbb{C}$ e $\min \{\operatorname{Re}(a), \operatorname{Re}(b)\}>\gamma>0, c \neq 0,-1,-2, \ldots$ e $|\arg (-z)|<\pi$. O caminho de integração é tal que os pólos em $s=a+n$ e $s=b+n$ estejam separados daqueles em $s=-n$ com $n=0,1,2, \ldots$

Esta representação integral para a clássica função hipergeométrica, nada mais é que uma integral do tipo Mellin-Barnes.

\section{Função $H$ de Fox}

A função $H$, conforme introduzida por Fox em 1961 [13], é uma função especial que contempla, como casos particulares, várias funções especiais da Física-Matemática e que permite tratar diferentes problemas de maneira unificada. Apenas para mencionar, problemas envolvendo difusão anômala [11, 12, 23] e problemas advindos da física de partículas elementares [1] são tratados via função $H$ de Fox.

Existem compêndios onde a função $H$ de Fox é abordada, em particular, mencionamos o artigo [24] e os livros [16, 25] porém não é unanimidade a maneira de introduzi-la no sentido de considerarmos, ou não, a transformada de Mellin, conforme anteriormente mencionado.

Neste trabalho, como já afirmamos, vamos introduzir a função $H$ de Fox através de uma integral de Mellin-Barnes de modo que podemos, quando necessário, interpretá-la como uma transformada de Mellin inversa. 
Consideramos os inteiros $m, n, p, q$ de modo que $0 \leq n \leq p$ e $1 \leq m \leq q$ e os parâmetros $a_{\ell}, b_{j} \in \mathbb{C}$ e $A_{\ell}, B_{j} \in \mathbb{R}_{+}$para $\ell=1, \ldots, p$ e $j=1, \ldots, q$. Definimos a função $H \equiv H(z)$ de Fox a partir da seguinte integral de Mellin-Barnes

$$
\begin{gathered}
H(z) \equiv H_{p, q}^{m, n}(z)=H_{p, q}^{m, n}\left[z \mid \begin{array}{c}
\left(a_{\ell}, A_{\ell}\right)_{1, p} \\
\left(b_{j}, B_{j}\right)_{1, q}
\end{array}\right]= \\
H_{p, q}^{m, n}\left[z \mid \begin{array}{c}
\left(a_{1}, A_{1}\right), \ldots,\left(a_{p}, A_{p}\right) \\
\left(b_{1}, B_{1}\right), \ldots,\left(b_{q}, B_{q}\right)
\end{array}\right]=\frac{1}{2 \pi i} \int_{\mathcal{L}} \mathcal{H}_{p, q}^{m, n}(s) z^{-s} \mathrm{~d} s,
\end{gathered}
$$

onde

$$
\mathcal{H}_{p, q}^{m, n}(s)=\frac{\prod_{j=1}^{m} \Gamma\left(b_{j}+B_{j} s\right) \prod_{\ell=1}^{n} \Gamma\left(1-a_{\ell}-A_{\ell} s\right)}{\prod_{\ell=n+1}^{p} \Gamma\left(a_{\ell}+A_{\ell} s\right) \prod_{j=m+1}^{q} \Gamma\left(1-b_{j}-B_{j} s\right)} .
$$

O contorno $\mathcal{L}$ na Eq.(3.1) pode ser escolhido de três maneiras distintas [16] porém para todas elas devemos impor que os pólos

$$
\begin{array}{lll}
b_{j \ell}=-\frac{b_{j}+\ell}{B_{j}} \quad \text { com } \quad j=1, \ldots, m ; & \ell=0,1,2, \ldots \\
a_{r k}=\frac{1-a_{r}+k}{A_{r}} & \text { com } \quad r=1, \ldots, n ; & k=0,1,2, \ldots
\end{array}
$$

não coincidam, isto é, os parâmetros complexos $a_{i}$ e $b_{i}$ são tomados com a imposição de que nenhum pólo do integrando venha a coincidir. O contorno dispõe todos os pólos em $s=b_{j \ell}$ à esquerda e todos os pólos $s=a_{r k}$ à direita de $\mathcal{L}$ [16]. Nos casos em que $n=0, m=q$ e $n=p$, devemos interpretar os produtórios como sendo 1 .

No particular caso em que $A_{\ell}=1=B_{j}$, para todo $j$ e $\ell$, recuperamos a chamada função $G$ de Meijer [27], isto é,

$$
H_{p, q}^{m, n}\left[z \mid \begin{array}{c}
\left(a_{\ell}, 1\right)_{1, p} \\
\left(b_{j}, 1\right)_{1, q}
\end{array}\right] \equiv G_{p, q}^{m, n}\left[z \mid \begin{array}{c}
\left(a_{\ell}\right)_{1, p} \\
\left(b_{j}\right)_{1, q}
\end{array}\right] .
$$

Braaksma [2] mostrou que, independentemente da escolha do contorno, a integral de Mellin-Barnes faz sentido e define uma função analítica na variável $z$ nos dois casos a seguir:

$$
\mu>0, \quad 0<|z|<\infty \quad \text { onde } \quad \mu=\sum_{j=1}^{q} B_{j}-\sum_{j=1}^{p} A_{j}
$$

$\mathrm{e}$

$$
\mu=0, \quad 0<|z|<\delta \quad \text { onde } \quad \delta=\prod_{j=1}^{p} A_{j}^{-A_{j}} \cdot \prod_{j=1}^{q} B_{j}^{B_{j}} .
$$




\subsection{Propriedades}

Como já mencionamos, vamos apresentar apenas as propriedades envolvendo a função $H$ de Fox que serão utilizadas na Seção 5.

\section{P.1. Mudança na variável independente}

Seja $c$ uma constante positiva. Temos

$$
H_{p, q}^{m, n}\left(\begin{array}{l|l}
x & \begin{array}{c}
\left(a_{p}, A_{p}\right) \\
\left(b_{q}, B_{q}\right)
\end{array}
\end{array}\right)=c H_{p, q}^{m, n}\left(\begin{array}{l|l}
x^{c} & \left(a_{p}, c A_{p}\right) \\
\left(b_{q}, c B_{q}\right)
\end{array}\right) .
$$

Para mostrar esta igualdade, basta introduzir a mudança de variável $s \rightarrow c s$ na integral da transformada de Mellin inversa.

\section{P.2. Mudança do primeiro argumento}

Seja $\alpha \in \mathbb{R}$. Então podemos escrever

$$
x^{\alpha} H_{p, q}^{m, n}\left(x \mid \begin{array}{c}
\left(a_{p}, A_{p}\right) \\
\left(b_{q}, B_{q}\right)
\end{array}\right)=H_{p, q}^{m, n}\left(\begin{array}{l|l}
x & \begin{array}{c}
\left(a_{p}+\alpha A_{p}, A_{p}\right) \\
\left(b_{q}+\alpha B_{q}, B_{q}\right)
\end{array}
\end{array}\right) .
$$

Para mostrar esta igualdade, introduzimos a mudança de variável $a_{p} \rightarrow a_{p}+\alpha A_{p}$ e tomamos $s \rightarrow s-\alpha$ na integral da transformada de Mellin inversa.

\section{P.3. Redução de ordem}

Se o primeiro fator $\left(a_{1}, A_{1}\right)$ é igual ao último, $\left(b_{q}, B_{q}\right)$, temos

$$
\begin{aligned}
H_{p, q}^{m, n}\left(x \mid \begin{array}{l}
\left(a_{1}, A_{1}\right), \ldots,\left(a_{p}, A_{p}\right) \\
\left(b_{1}, B_{1}\right), \ldots,\left(b_{q-1}, B_{q-1}\right)\left(a_{1}, A_{1}\right)
\end{array}\right)= \\
=H_{p-1, q-1}^{m, n-1}\left(x \mid \begin{array}{l}
\left(a_{2}, A_{2}\right), \ldots,\left(a_{p}, A_{p}\right) \\
\left(b_{1}, B_{1}\right), \ldots,\left(b_{q-1}, B_{q-1}\right)
\end{array}\right) .
\end{aligned}
$$

Para mostrar esta identidade é suficiente simplificar os argumentos comuns na integral de Mellin-Barnes.

\section{Cálculo Fracionário}

O cálculo fracionário é uma das ferramentas mais precisas para se refinar a descrição de fenômenos naturais. Uma maneira bastante comum de se utilizar esta ferramenta é substituir a derivada de ordem inteira de uma equação diferencial parcial, que descreve um determinado fenômeno, por uma derivada de ordem não inteira. Vários resultados importantes e generalizações foram obtidos através desta técnica, em diversas áreas do conhecimento, tais como: mecânica dos fluidos, fenômenos de transporte, redes elétricas, probabilidade, biomatemática, dentre outros [8].

Para resolver a equação diferencial parcial fracionária, utilizamos a metodologia da justaposição de transformadas, ou seja, aplicamos a transformada de Fourier na 
parte espacial e a transformada de Laplace para eliminar a dependência temporal. Sendo assim, nesta seção apresentamos a chamada derivada fracionária no sentido de Caputo [6], bem como suas transformadas de Laplace e Fourier. Além disso, recuperamos alguns resultados envolvendo as funções de Mittag-Leffler [21].

\subsection{Derivada de Ordem Não Inteira}

Há várias formas de se introduzir a derivada de ordem não inteira como uma generalização para a derivada de ordem inteira, dentre elas podemos citar a definição de Riemann-Liouville, que é a mais conhecida e a de Caputo, que é mais restritiva, mas parece ser mais adequada para o estudo de problemas físicos [10]. A derivada de ordem $\mu$ no sentido de Caputo é definida da seguinte maneira [6]

$$
\mathrm{D}_{t}^{\mu} f(t, x) \equiv \frac{\partial^{\mu}}{\partial t^{\mu}} f(t, x)= \begin{cases}\frac{1}{\Gamma(n-\mu)} \int_{a}^{t} \frac{f^{(n)}(\tau, x)}{(t-\tau)^{\mu+1-n}} \mathrm{~d} \tau, & n-1<\mu<n, \\ f^{(n)}(t, x), & \mu \equiv n \in \mathbb{N}\end{cases}
$$

na qual $f^{(n)}(t, x)$ denota a derivada usual de ordem $n$ em relação à variável $t$.

Deste ponto em diante, consideramos o limite inferior $a$ como sendo $-\infty$ na parte espacial e zero na parte temporal. O primeiro e segundo casos estão associados, respectivamente, às transformadas de Fourier e de Laplace [20].

Sendo $s, \operatorname{com} \operatorname{Re}(s)>0$, o parâmetro da transformada de Laplace temos [30]

$$
\mathfrak{L}\left\{\frac{\partial^{\mu}}{\partial t^{\mu}} f(t, x)\right\}=s^{\mu} F(s, x)-\sum_{k=0}^{n-1} s^{\mu-1-k} f^{(k)}\left(0^{+}, x\right)
$$

com $n-1<\mu \leq n$ e $n \in \mathbb{N}$. Nesta equação, $F(s, x)$ denota a transformada de Laplace de $f(t, x)$. Além disso, sendo $\omega$ o parâmetro da transformada de Fourier podemos escrever para a derivada fracionária de Caputo

$$
\mathfrak{F}\left\{\mathrm{D}_{x}^{\mu} f(t, x)\right\}=|\omega|^{2 \mu} F(t, \omega),
$$

na qual $F(t, \omega)$ é a transformada de Fourier da função $f(t, x)$.

Enquanto a transformada de Laplace da derivada fracionária de Caputo depende de condições iniciais que possuem uma imediata interpretação física, a derivada fracionária segundo Riemann-Liouville depende de condições dadas em termos de

$\left.{ }_{a} \mathrm{D}_{t}^{\mu-k-1} f(t)\right|_{t=0}$. Outra importante diferença entre estas duas abordagens é que a derivada fracionária de Caputo de uma constante é zero, o que não ocorre com a definição de Riemann-Liouville. ${ }^{9}$

\subsection{Funções de Mittag-Leffler}

Nesta seção introduzimos a clássica função de Mittag-Leffler, denotada por $E_{\alpha}(x)$, bem como a função de Mittag-Leffler de dois parâmetros, denotada por $E_{\alpha, \beta}(x)$, a

\footnotetext{
${ }^{9}$ Note que desta forma a derivada segundo Riemann-Liouville não pode ser interpretada como a taxa de variação. Isto justifica a utilização da derivada de Caputo e não a de Riemann-Liouville, quando estamos interessados em resolver uma equação diferencial parcial fracionária.
} 
partir da função de Mittag-Leffler com três parâmetros, também conhecida como função de Mittag-Leffler generalizada, proposta por Prabhakar [31], isto é,

$$
E_{\alpha, \beta}^{\rho}(z)=\sum_{k=0}^{\infty} \frac{(\rho)_{k}}{\Gamma(k \alpha+\beta)} \frac{z^{k}}{k !} \equiv \frac{1}{\Gamma(\rho)} H_{1,2}^{1,1}\left(-z \mid \begin{array}{l}
(1-\rho, 1) \\
(0,1),(1-\beta, \alpha)
\end{array}\right)
$$

na qual $(\rho)_{k}$ é o símbolo de Pochhammer,

$$
(\rho)_{k}=\frac{\Gamma(\rho+k)}{\Gamma(\rho)} \equiv \rho(\rho+1) \cdots(\rho+k-1)
$$

e $z \in \mathbb{C}, \operatorname{Re}(\rho)>0, \operatorname{Re}(\alpha)>0$ e $\operatorname{Re}(\beta)>0$. Esta função generaliza a função de Mittag-Leffler clássica e também a de dois parâmetros [22], pois

$$
E_{\alpha, 1}^{1}(x)=E_{\alpha}(x)=\sum_{k=0}^{\infty} \frac{z^{k}}{\Gamma(k \alpha+1)} \quad \text { e } \quad E_{\alpha, \beta}^{1}(x)=E_{\alpha, \beta}(x)=\sum_{k=0}^{\infty} \frac{z^{k}}{\Gamma(k \alpha+\beta)},
$$

consequentemente para $\alpha, \beta, \rho=1$ temos $E_{1,1}^{1}(x)=\mathrm{e}^{x}$.

Podemos escrever a transformada de Laplace da função de Mittag-Leffler com três parâmetros [16], ou seja,

$$
\mathfrak{L}\left[t^{\beta-1} E_{\alpha, \beta}^{\rho}\left( \pm \lambda t^{\alpha}\right)\right]=\frac{s^{\alpha \rho-\beta}}{\left(s^{\alpha} \mp \lambda\right)^{\rho}}
$$

cuja transformada inversa pode ser escrita da seguinte forma

$$
\mathfrak{L}^{-1}\left[\frac{s^{\alpha \rho-\beta}}{\left(s^{\alpha} \mp \lambda\right)^{\rho}}\right]=t^{\beta-1} E_{\alpha, \beta}^{\rho}\left( \pm \lambda t^{\alpha}\right),
$$

com $\operatorname{Re}(\alpha)>0$ e $\operatorname{Re}(\beta)>0$.

Por conveniência, no que se segue, vamos introduzir a seguinte função do tipo Mittag-Leffler

$$
\mathcal{E}_{\alpha, \beta}^{\rho}(t, y, \gamma) \equiv t^{\beta-1} E_{\alpha, \beta}^{\rho}\left(-\mathcal{K}|y|^{\gamma} t^{\alpha}\right),
$$

satisfazendo $\operatorname{Re}(\alpha)>0, \operatorname{Re}(\beta)>0, \operatorname{Re}(\rho)>0, \mathcal{K}$ é uma constante positiva e $y=\left(y_{1}, y_{2}, \cdots, y_{n}\right) \in \mathbb{R}^{n}$. Aqui, associamos a variável $t$ como sendo o tempo enquanto que a variável $y$ como sendo a variável espacial. Note-se que, no caso em que $\rho=1$, recuperamos os recentes resultados obtidos por Yu \& Zhang [35].

\section{A Equação do Telégrafo Fracionária}

Como aplicação, discutimos a equação do telégrafo fracionária no caso tridimensional, isto é, a dimensão espacial igual a três.

A equação diferencial parcial fracionária a ser estudada é

$$
a \mathrm{D}_{t}^{2 \alpha} u+b \mathrm{D}_{t}^{\beta} u=-\mathcal{K}(-\Delta)^{\gamma} u, \quad t>0 ; \quad x \in \mathbb{R}^{n} ;
$$

$\operatorname{com} \mathrm{D} \equiv \partial / \partial t, 1 / 2<\alpha \leq 1,0<\beta \leq 1$ e $0<\gamma \leq 1$, com $u=u(t, x ; \alpha, \beta, \gamma)$ e $x=x\left(x_{1}, x_{2}, x_{3}\right)$. Aqui $(-\Delta)^{\gamma}$ denota o operador de Laplace fracionário [7]. 
$a, b \in \mathbb{R}, \mathcal{K}$ uma constante física real, $t$ é a variável temporal e $x$ é a variável espacial. No caso em que $\alpha=\beta=\gamma=1$ recuperamos a clássica equação do telégrafo. Assim a Eq.(5.1) pode ser considerada como uma generalização da clássica equação do telégrafo. Aqui, não estamos preocupados com as unidades físicas [15].

Admitamos as seguintes condições iniciais e de contorno

$$
\lim _{|x| \rightarrow \infty} u(t, x ; \alpha, \beta, \gamma)=0 \quad \text { e } \quad \mathrm{D}_{t}^{k} u(t, x ; \alpha, \beta, \gamma)=f_{k}(x),
$$

respectivamente, para $k=0,1, \ldots, m-1$.

Enfim, consideremos a derivada fracionária temporal no sentido de Caputoa derivada espacial fracionária é no sentido de Riesz e usamos a relação [7]

$$
\mathfrak{F}\left[(-\Delta)^{\gamma} u(t, x ; \alpha, \beta, \gamma) ; \omega\right]=|\omega|^{2 \gamma} \mathfrak{F}[u(t, x ; \alpha, \beta, \gamma) ; \omega],
$$

onde $\omega$ é o parâmetro associado à transformada de Fourier, de onde obtemos uma outra equação diferencial fracionária

$$
a \mathrm{D}_{t}^{2 \alpha} \widehat{u}+b \mathrm{D}_{t}^{\beta} \widehat{u}=-\mathcal{K}|\omega|^{2 \gamma} \widehat{u}
$$

satisfazendo as condições iniciais $\mathrm{D}_{t}^{k} \widehat{u}(t, \omega, \alpha, \beta, \gamma)=F_{k}(\omega) \operatorname{com} k=0,1, \ldots, m-1$, onde $\widehat{u}=\widehat{u}(t, \omega ; \alpha, \beta, \gamma)$ é a transformada de Fourier de $u=u(t, x ; \alpha, \beta, \gamma)$ e $F_{k}(\omega)$ é a transformada de Fourier de $f_{k}(x)$.

Como já afirmamos, consideramos apenas o caso $n=3$. Então, tomando a transformada de Laplace da Eq.(5.2) e usando as condições iniciais, obtemos uma equação algébrica cuja solução é dada por

$$
\widehat{u}(p, \omega ; \alpha, \beta, \gamma)=F_{0}(\omega) \frac{a p^{2 \alpha-1}+b p^{\beta-1}}{a p^{2 \alpha}+b p^{\beta}+\mathcal{K}|\omega|^{2 \gamma}},
$$

onde $p$ é o parâmetro da transformada de Laplace e $\widehat{u}(p, \omega ; \alpha, \beta, \gamma)$ é a justaposição da transformada inversa de $u(t, x ; \alpha, \beta, \gamma)$. Para obter a última expressão usamos as condições

$$
u(0, x ; \alpha, \beta, \gamma)=f_{0}(x) \quad \Longleftrightarrow \quad \widehat{u}(0, \omega ; \alpha, \beta, \gamma)=F_{0}(\omega)
$$

$\mathrm{e}$

$$
\left.\frac{\partial}{\partial t} u(t, x ; \alpha, \beta, \gamma)\right|_{t=0}=f_{1}(x)=\left.0 \quad \Longleftrightarrow \quad \frac{\partial}{\partial t} \widehat{u}(t, \omega ; \alpha, \beta, \gamma)\right|_{t=0}=F_{1}(\omega)=0 .
$$

Assim, devemos proceder com a inversão. Tomando a correspondente transformada de Laplace inversa, podemos escrever [11]

$$
\begin{aligned}
& \widehat{u}(t, \omega ; \alpha, \beta, \gamma)=F_{0}(\omega) \sum_{r=0}^{\infty}\left(-\frac{b}{a}\right)^{r} t^{(2 \alpha-\beta) r} E_{2 \alpha,(2 \alpha-\beta) r+1}^{r+1}\left(-\frac{\mathcal{K}}{a}|\omega|^{2 \gamma} t^{2 \alpha}\right) \\
& +\frac{b}{a} F_{0}(\omega) \sum_{r=0}^{\infty}\left(-\frac{b}{a}\right)^{r} t^{(2 \alpha-\beta)(r+1)} E_{2 \alpha,(2 \alpha-\beta)(r+1)+1}^{r+1}\left(-\frac{\mathcal{K}}{a}|\omega|^{2 \gamma} t^{2 \alpha}\right)
\end{aligned}
$$


$\operatorname{com} \operatorname{Re}(\alpha)>1 / 2, \operatorname{Re}(\beta)>0, \operatorname{Re}(s)>0, \alpha>\beta,\left|b s^{\beta} /\left(a s^{\alpha}+\mathcal{K}|\omega|^{2 \gamma}\right)\right|<1$ e $E_{\alpha, \beta}^{\gamma}(z)$ é a função de Mittag-Leffler generalizada.

Usando a Eq.(4.1) podemos reescrever a Eq.(5.3) na forma

$$
\widehat{u}(t, \omega ; \alpha, \beta, \gamma)=F_{0}(\omega) \sum_{r=0}^{\infty}\left(-\frac{b}{a}\right)^{r}\left\{\mathcal{E}_{2 \alpha, \mu r+1}^{r+1}(t, \omega ; \gamma)+\frac{b}{a} \mathcal{E}_{2 \alpha, \mu(r+1)+1}^{r+1}(t, \omega ; \gamma)\right\}
$$

onde definimos o parâmetro $\mu=2 \alpha-\beta$.

Para calcular a respectiva transformada de Fourier inversa, levamos em conta o teorema de convolução, logo,

$$
u(t, x ; \alpha, \beta, \gamma) \equiv \mathfrak{F}^{-1}[\widehat{u}(t, \omega ; \alpha, \beta, \gamma) ; \omega]=\sum_{r=0}^{\infty}\left(-\frac{b}{a}\right)^{r} \int_{\mathbb{R}^{3}} F_{0}(\xi) \mathcal{G}(t ; x-\xi) \mathrm{d} \xi,
$$

onde a função $\mathcal{G}(t, x)$, como no caso inteiro, é conhecida como solução fundamental, que é dada por

$$
\mathcal{G}(t ; x)=\widehat{\mathcal{E}}_{2 \alpha, \mu r+1}^{r+1}(t, x ; \gamma)+\frac{b}{a} \widehat{\mathcal{E}}_{2 \alpha, \mu(r+1)+1}^{r+1}(t, x ; \gamma),
$$

a qual pode ser escrita em termos da função $H$ de Fox, como abaixo

$$
u(t, x ; \alpha, \beta, \gamma)=\frac{1}{(\sqrt{\pi}|x|)^{3}} \sum_{r=0}^{\infty}\left(-\frac{b}{a}\right)^{r} \frac{t^{\mu r}}{r !} \int_{\mathbb{R}^{3}} F_{0}(\xi) \mathfrak{H}_{2,3}^{2,1}(x-\xi) \mathrm{d} \xi,
$$

onde definimos

$$
\begin{aligned}
\mathfrak{H}_{2,3}^{2,1}(x)=\quad H_{2,3}^{2,1}\left(\frac{|x|^{2 \gamma}}{2^{2 \gamma} \mathcal{K} t^{2 \alpha}} \mid \begin{array}{l}
(1,1),(\mu r+1,2 \alpha) \\
(r+1,1),\left(\frac{3}{2}, \gamma\right),(1, \gamma)
\end{array}\right) & +\frac{b}{a} t^{\mu} H_{2,3}^{2,1}\left(\frac{|x|^{2 \gamma}}{2^{2 \gamma} \mathcal{K} t^{2 \alpha}} \begin{array}{l}
(1,1),[\mu(r+1)+1,2 \alpha] \\
(r+1,1),\left(\frac{3}{2}, \gamma\right),(1, \gamma)
\end{array}\right),
\end{aligned}
$$

$\operatorname{com} \mu=2 \alpha-\beta$.

\section{Conclusões}

A partir do conceito de integrais de Mellin-Barnes, introduzimos a função $H$ de Fox e apresentamos algumas de suas propriedades. Como caso particular da função $H$ de Fox, mencionamos a função de Mittag-Leffler. Como uma aplicação discutimos a equação diferencial fracionária associada ao problema do telégrafo utilizando a definição da derivada fracionária no sentido de Caputo. A solução desta equação diferencial fracionária foi apresentada em termos de uma função $H$ de Fox.

\section{Agradecimentos}

Somos gratos ao referee pelas sugestões que contribuíram para a melhora do texto.

Abstract. Using the concept of Mellin-Barnes integral, we present the Fox's $H$ function and discuss some of them properties to discuss the so-called fractional telegraph equation, i.e., the fractional partial differential equation associated with the telegraph problem, whose solution was presented in terms of the Fox's $H$ function. 


\section{Referências}

[1] R.F. Ávila, M.J. Menon, Eikonal zeros in the momentum transfer space from proton-proton scattering: An empirical analysis, Eur. Phys. J., 54C (2008), $555-576$.

[2] B.L.J. Braaksma, Asymptotic expansions and analytical continuation for a class of Barnes-integrals, Compositio Math., 15 (1962), 239-341.

[3] E. Capelas de Oliveira, "Funções Especiais com Aplicações", Editora Livraria da Física, São Paulo, 2005.

[4] E. Capelas de Oliveira, J. Vaz Jr., FS. Costa, The fractional Schrödinger equation for delta potentials, (2010), Journal of Mathematical Physics (aceito).

[5] E. Capelas de Oliveira, J. Vaz Jr., Tunneling in fractional quantum mechanics, (2010) Submetido à publicação.

[6] M. Caputo, Vibrations of an infinite viscoelastic layer with a dissipative memory, J. Acoust. Soc. Amer., 56 (1974), 897-904.

[7] W. Chen, S. Holm, Physical interpretation of fractional diffusion-wave equation via lossy media obeying frequency power law, arXiv.org/abs/math-ph/0303040 (2003).

[8] Debnath, Recent applications of fractional calculus to science and engineering, Int. J. Math., 2003 (2003), 3413-3442.

[9] A. Erdélyi, W. Magnus, F. Oberhettinger, F.G. Tricomi, "Higher Transcendental Functions", Vol.1, McGraw-Hill, New York, 1953.

[10] R. Figueiredo Camargo, A.O. Chiacchio, E. Capelas de Oliveira, Differentiation to fractional orders and the fractional telegraph equation, J. Math. Phys., 49 (2008), 033505.

[11] R. Figueiredo Camargo, R. Charnet, E. Capelas de Oliveira, On some fractional Green's functions, J. Math. Phys., 50 (2009), 043514.

[12] R. Figueiredo Camargo, E. Capelas de Oliveira, J. Vaz Jr., On anomalous diffusion and the fractional generalized Langevin equation for a harmonic oscillator, J. Math. Phys., 50 (2009), 123518.

[13] C. Fox, The $G$ and $H$ functions as symmetrical Fourier kernels, Trans. Amer. Math. Soc., 98 (1961), 395-429.

[14] D. Gomes, E. Capelas de Oliveira, The generating function for $E_{n}^{\ell}(\rho)$ polynomials, Algebras, Groups and Geometries, 14 (1997) 49-57.

[15] P. Inizan, Homogeneous fractional embeddings, J. Math. Phys., 49 (2008), 082901. 
[16] A.A. Kilbas, H.M. Srivastava, J.J. Trujillo, "Theory and Applications of Fractional Differential Equations", Mathematics Studies, Vol. 204, Edited by Jan van Mill, Elsevier, Amsterdam, 2006.

[17] V. Kiryakova, The special functions of fractional calculus as generalized fractional calculus operators of some basic functions, Comp. Math. Appl., 59 (2010), $1128-1141$.

[18] G. Lauricella, Sulla funzione ipergeometrica a più variabili, Rend. Circ. Math. Palermo, 7 (1893), 111-158.

[19] E.K. Lenzi, L.R. Evangelista, M. K. Lenzi, H. V. Ribeiro and E. Capelas de Oliveira, Solutions of a Non-Markovian Diffusion Equation, Phys. Lett. A, 374 (2010) 4193-4198.

[20] C.F. Lorenzo, T.T. Hartley, Initialized fractional calculus, NASA/TP-2000209943.

[21] F. Mainardi, "Fractional Calculus and Waves in Linear Viscoelasticity", World Scientific Publishing Co., London, 2010.

[22] F. Mainardi, R. Gorenflo, On Mittag-Leffler-type functions in fractional evolution process, J. Comput. Appl. Math., 118 (2000), 283-299.

[23] F. Mainardi, G. Pagnini, Salvatore Pincherle: the pioneer of the Mellin-Barnes integrals, J. Comput. Appl. Math., 153 (2003), 331-342.

[24] F. Mainardi, G. Pagnini, R.K. Saxena, Fox $H$ function in fractional diffusion, J. Comput. Appl. Math., 178 (2005), 321-331.

[25] A.M. Mathai, H.J. Haubold, "Special Functions for Applied Scientistic", Springer, Heidelberg, 2008.

[26] A.M. Mathai, R.S. Saxena, H.J. Haubold, "The $H$-Function. Theory and Applications", Springer, New York, 2010.

[27] G.S. Meijer, On the $G$ function I-VIII, Nederl. Akad. Wettensch. Proc., 49, (1946), 227-237, 344-356, 457-469, 632-641, 765-772, 936-943, 1063-1072, 1165-1175. Traduzido para o Inglês: Indag. Math., 8, (1946), 124-134, 213$225,312-324,391-400,468-475,595-602,661-670$ e $713-723$.

[28] E.A. Notte Cuello, M.J. Menon, E. Capelas de Oliveira, Inverse problems in Hadron scattering and the Fox's $H$-function (2010), Submetido à publicação.

[29] S. Pincherle, Sulle funzioni ipergeometriche generalizzate, Atti R. Accad. Lincei, Rend. Cl. Sci. Fis. Mat. Natur., 4 (1888), 694-700 e 792-799.

[30] I. Podlubny, "Fractional Differential Equations", Mathematics in Science and Engineering, Vol.198, Academic Press, San Diego, 1999.

[31] T.R. Prabhakar, A singular integral equation with generalized Mittag-Leffler function in the kernel, Yokohama Math. J., 19 (1971), 7-15. 
[32] E.D. Rainville, "Special Function", The Macmillan Company, New York, 1967.

[33] R.K. Saxena, In memorium of Charles Fox, Fract. Cal. Appl. Anal., 12 (2009), $337-344$.

[34] I.N. Sneddon, "The Use of Integral Transforms", McGraw-Hill, New York, 1972.

[35] Rui Yu, H. Zhang, New function of Mittag-Leffler type and its application in the fractional diffusion-wave equation, Chaos, Solitons \& Fractals, 30 (2006), 946-955.

[36] N.N. Temme, "Special Function: An Introduction to the Classical Functions of Mathematical Physics", Wiley, New York, 1996. 